\title{
Competition between elastic and chemical effects in the intermixing of Co and Ag on $\operatorname{Rh}(111)^{\dagger}$
}

\author{
MIGHFAR IMAM, MADHURA MARATHE and SHOBHANA NARASIMHAN* \\ Theoretical Sciences Unit, Jawaharlal Nehru Centre for Advanced Scientific Research, \\ Jakkur Campus, Bangalore 560064 \\ e-mail: shobhana@jncasr.ac.in
}

\begin{abstract}
We have performed ab initio density functional theory calculations to investigate the formation energy and the effects of low dimensionality and reduced coordination on the magnetic properties of pseudomorphically grown monolayers of $\mathrm{Co}-\mathrm{Ag}$ surface alloys on a $\mathrm{Rh}(111)$ substrate. We find that if such an alloy could form, its magnetic moment would be considerably enhanced relative to that of bulk Co. However, we also find that its formation is energetically disfavoured against phase-segregated forms; this can be mainly attributed to the high 'chemical' cost of forming Ag-Co bonds. Trends in the variation of magnetic moment with alloy composition are largely consistent with the Stoner argument.
\end{abstract}

Keywords. Surface alloys; reduced coordination; magnetism; strain.

\section{Introduction}

Alloying phenomena at surfaces can drastically differ from those in the bulk. The observed alloying of metals at surfaces which are immiscible in the bulk has attracted much attention. ${ }^{1-4}$ Apart from the intermixing of adsorbate and substrate to form a thin film alloy, ${ }^{5}$ quasi two-dimensional nanoscale patterns formed by the deposition of two bulk-immiscible metals upon a suitable substrate are of particular interest. $^{6-8}$ For some applications, it is desirable to form a stable or quasi-stable pattern of domains of a magnetic material separated by spacers of non-magnetic material. However, mixing at the atomic level may also confer beneficial properties, for example, it may increase magnetic anisotropy energies, or even, in some cases, contribute to increased magnetic moments. For applications in industry, it is desirable to develop novel magnetic materials with high magnetic moments and anisotropies, so that information storage is not degraded by the thermal flipping of spins.

From a general point of view, it is also of interest to see whether bulk-immiscible metals can be forced to alloy in two-dimensions, by depositing them on a substrate of intermediate lattice spacing. Co and $\mathrm{Ag}$ do not alloy in the bulk, because of the large size mismatch between them. The nearest-neighbour (NN)

${ }^{\dagger}$ Dedicated to Prof. C N R Rao on his 75th birthday

*For correspondence distance is $2.51 \AA$ in Co and $2.89 \AA$ in Ag. However, the mean of these two values is $2.70 \AA$, which matches very well with the $\mathrm{Ru} \mathrm{NN}$ distance of $2.74 \AA$. It therefore might seem, at first sight, reasonable to expect that the strain imposed by a $\mathrm{Ru}(0001)$ substrate would favour the surface alloying of $\mathrm{Ag}$ and $\mathrm{Co}$. However, experiments ${ }^{9}$ on this system found that there was no atomic-level intermixing in this system. Instead, an irregular network of $\mathrm{Co}$ and $\mathrm{Ag}$ islands was observed. By performing calculations, it was deduced that this may be attributable to chemical-bonding effects. ${ }^{10}$ An alternative explanation is also possible: that effective atomic sizes on surfaces differ significantly from those in the bulk. If the atomic sizes of $\mathrm{Ag}$ and $\mathrm{Co}$ on substrate were to be significantly lower than the corresponding bulk values, then even strain effects may not favour alloying. To further examine this question, as well as to check what difference, if any, is made by changing the substrate, in this paper we look at $\mathrm{Ag}$ and Co co-deposited on a $\mathrm{Rh}(111)$ substrate. The Rh NN spacing is $2.69 \AA$, which would again suggest, applying the simple criterion mentioned above, that elastic effects should favour alloying.

\section{Method}

Our $a b$ initio calculations were performed using the Plane Wave Self-Consistent Field (PWscf) code within the framework of Density Functional Theory 
(DFT). ${ }^{11}$ The interaction between ions and valence electrons for all metals was described by an ultrasoft (Vanderbilt) pseudopotential. ${ }^{12}$ The Perdew-BurkeErnzerhof (PBE) form of the generalized gradient approximation (GGA) ${ }^{13}$ for the exchange-correlation functional was used. The Methfessel-Paxton smearing scheme ${ }^{14}$ was used, with smearing parameter set equal to $0.05 \mathrm{Ry}$. All the structures were relaxed using Hellmann-Feynman Forces. ${ }^{15}$

The equilibrium lattice constants of $\mathrm{Co}, \mathrm{Ag}$ and $\mathrm{Rh}$ in their respective bulk structures were determined by performing a series of self consistent total energy calculations and fitting the data to the Birch second order equation of state. ${ }^{16}$ For all bulk calculations, a plane wave basis set-up to a kinetic energy cut-off of $20 \mathrm{Ry}(272 \mathrm{eV})$ was used, together with a kinetic energy cut-off for the charge density of $160 \mathrm{Ry}$. Brillouin zone integrations made use of a $8 \times 8 \times 8$ Monkhorst-Pack grid.

We considered binary surface alloys of Co and $\mathrm{Ag}$, deposited pseudomorphically on the $\mathrm{Rh}$ (111) substrate. The substrate consisted of a $2 \times 2$ surface unit cell with six layers of $\mathrm{Rh}(111)$. This surface cell enabled us to consider surface alloys of the form $\mathrm{Co}_{x} \mathrm{Ag}_{1-x}$, with $x=0.0,0.25,0.5,0.75$ and 1.0 . With this surface cell, only one inequivalent configuration is possible for each value of $x$. As an example, we show in figure 1, a top view of the configuration for $x=0.5$. Each of the four overlayer atoms can be

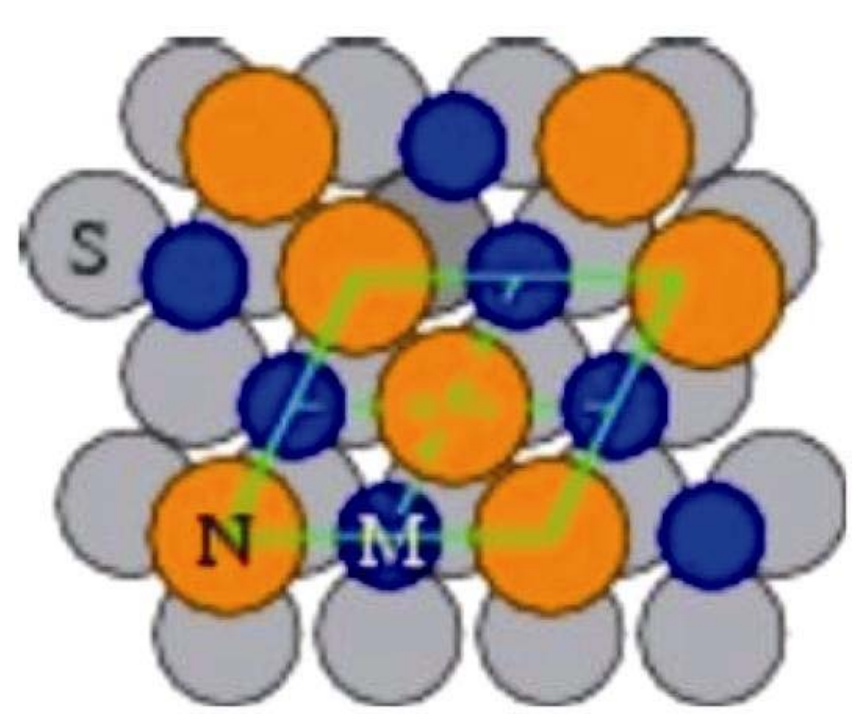

Figure 1. Top view of surface slab for $x=0 \cdot 5$ : the rhombus indicates the $2 \times 2$ surface unit cell, and blue, orange and grey circles represent $\mathrm{Co}, \mathrm{Ag}$ and $\mathrm{Rh}$ atoms respectively. The overlayer Ag and Co atoms can occupy either $f c c$ or $h c p$ sites relative to the substrate. placed on either the four face-centered cubic $(f c c)$ or four hexagonal closed packing ( $h c p$ ) sites available in this cell.

Periodic images were separated by a vacuum of about $17 \AA$ along the $z$ ([111]) direction. The top four (three of $\mathrm{Rh}$ and one of alloy) layers were allowed to relax and the bottom three were kept fixed at the bulk separation of the substrate. For the surface calculations, we used a $4 \times 4 \times 1 k$-point mesh for Brillouin zone integration. We performed checks for convergence with respect to $k$-points and the energy cut-off, for both bulk and slab structures.

All calculations were performed using the spinpolarized version of the Kohn-Sham equations, so as to enable us to study magnetic effects. We found that the incorporation of magnetic effects is crucial even in determining energetics accurately.

\section{Results and discussion}

\subsection{Bulk}

Bulk nearest neighbour distances for each elemental metal (and, for $\mathrm{Co}$, the magnetic moment) were calculated in their respective equilibrium structures. For $\mathrm{Rh}$, these values were obtained not using the primitive $f c c$ cell but a unit cell consisting of three atoms stacked along the [111] direction. This cell is commensurate to that used for the surface alloy calculations.

Our calculated values for bulk NN distances are $2.73 \AA, 2.95 \AA$ and $2.52 \AA$ for $\mathrm{Rh}, \mathrm{Ag}$ and Co respectively, which are in good agreement with the experimental values $2.69 \AA, 2.89 \AA$ and $2.51 \AA^{17}$ The calculated magnetic moment for $\mathrm{Co}$, of $1.71 \mu_{B}$ per atom, is also in good agreement with the experimental value of $1.72 \mu_{B}{ }^{18}$

\section{$3.2 R h(111)$ slab}

We considered both nine-layer and six-layer $\mathrm{Rh}$ slabs in the [111] direction. We considered two cases: (1) a symmetric slab and (2) an asymmetric slab. In the six-layer symmetric case we allowed the two top and two bottom layers to relax, whereas in the nine-layer symmetric case we allowed the outermost three layers on both sides to relax. In the asymmetric case, only the corresponding numbers of top layers were relaxed. It was found that all the layers which were free to move relaxed inward in all the cases. This inward relaxation was similar in the 
asymmetric and symmetric cases: for the asymmetric case, the inward relaxation was $\Delta_{12}=2.4 \%, \Delta_{23}=$ $1.3 \%$, and $\Delta_{34}=0.34 \%$, where $\Delta_{i j}$ is the percent change of the interlayer separation between the layers $i$ and $j$, relative to the bulk interlayer spacing. For the symmetric case, $\Delta_{12}=2.3 \%, \Delta_{23}=1.2 \%$, and $\Delta_{34}=0.29 \%$. We found the surface energy of the relaxed surface to be equal to $125.8 \mathrm{meV} / \AA^{2}$, which is in good agreement with an earlier calculation. ${ }^{19}$

\subsection{Monolayers on Rh (111)}

In our calculations of monolayers on $\mathrm{Rh}(111)$ we find that, as expected, a monolayer of Co tends to relax inward while that of $\mathrm{Ag}$ tends to move outward, relative to the interlayer separation in $\mathrm{Rh}(111)$. The overlayer-substrate interlayer distance is $2.42 \AA$ for $\mathrm{Ag} / \mathrm{Rh}(111)$ and $2.02 \AA$ for $\mathrm{Co} / \mathrm{Rh}(111)$.

We expect that the effective size of the overlayer atoms is different at the surface than in the bulk. In order to calculate the effective size of atoms at the surface, we compressed/stretched a symmetric slab containing $n_{a}$ layers (of which the outermost on both sides consisted of $\mathrm{Ag}$ or $\mathrm{Co}$, and the remaining layers consisted of Rh) in the $x y$ plane to a $\mathrm{NN}$ distance $l_{x y}$,

Table 1. Parameters deduced for a harmonic NN interaction for monolayers of $\mathrm{Ag}$ and $\mathrm{Co}$ on $\mathrm{Rh}(111): \sigma_{x x}^{\text {surf }}$ is the $a b$ initio value for the surface stress, $a$ is the NN distance in the bulk, $a^{s}$ is the surface atomic diameter (the optimal NN separation at the surface), and $k$ is the spring constant for NN bonds within the atomic monolayer.

\begin{tabular}{ccccc}
\hline & $a(\AA)$ & $\sigma_{x x}^{\text {surf }}\left(\mathrm{meV} / \AA^{2}\right)$ & $a^{s}(\AA)$ & $k\left(\mathrm{meV} / \AA^{2}\right)$ \\
\hline $\mathrm{Ag}$ & $2 \cdot 95$ & 222 & $2 \cdot 83$ & 3500 \\
$\mathrm{Co}$ & $2 \cdot 51$ & 620 & $2 \cdot 37$ & 2715 \\
\hline
\end{tabular}

Table 2. Formation energy and magnetic properties as a function of composition: $\Delta H / A$ is the formation energy per unit area of the surface alloy; $M_{C o}$ is the magnetic moment of an individual Co atom, while $M_{\text {tot }}$ includes the contribution (per Co atom) of induced moments on $\mathrm{Rh}$ and $\mathrm{Ag}$ atoms; $D O S_{C o}\left(E_{f}\right)$ and $D O S_{t o t}\left(E_{f}\right)$ are the density of states at the Fermi level for Co atoms and the whole system respectively.

\begin{tabular}{lccccc}
\hline & $\begin{array}{c}\Delta H / A \\
\left(\mathrm{meV} / \AA^{2}\right)\end{array}$ & $\begin{array}{c}M_{C o} \\
\left(\mu_{B}\right)\end{array}$ & $\begin{array}{c}D O S_{C o}\left(E_{f}\right) \\
(\text { states/eV) }\end{array}$ & $\begin{array}{c}M_{\text {tot }} \\
\left(\mu_{B}\right)\end{array}$ & $\begin{array}{c}D O S_{\text {tot }}\left(E_{f}\right) \\
(\text { states } / \mathrm{eV})\end{array}$ \\
\hline 0.25 & 6.80 & 1.91 & 4.39 & 3.03 & 47.81 \\
0.50 & 9.16 & 1.94 & 4.23 & 2.75 & 25.59 \\
0.75 & 6.42 & 1.96 & 4.34 & 2.81 & 18.33 \\
1.00 & 0.00 & 1.98 & 4.65 & 2.67 & 15.05 \\
\hline
\end{tabular}

while keeping the separation along the $z$ direction (surface normal) constant. The corresponding surface stress was then computed, using the equation:

$$
\begin{aligned}
\sigma_{x x}^{\text {surf }}\left(l_{x y}\right)= & \frac{1}{2}\left[\sigma_{x x}^{V, s l a b}\left(l_{x y}\right) L_{z}-\left(n_{a}-2\right)\right. \\
& \left.\sigma_{x x}^{b u l k}\left(l_{x y}\right) \frac{c}{3}+\sigma_{z z}^{b u l k}\left(l_{x y}\right) \frac{l_{x y}^{2}}{2 c}\right] .
\end{aligned}
$$

In this equation, the first term on the right-hand side is the 'volume' stress obtained for the slab from $a b$ initio calculations, i.e. it has dimensions of force per unit area, as opposed to surface stress, which has dimensions of force divided by length. The second and third terms are included to subtract out the contribution from the substrate layers; they involve the components of the stress tensor for bulk $\mathrm{Rh}$; these are determined by performing an additional series of calculations on a bulk $\mathrm{Rh}$ unit cell, consisting of three close packed layers stacked along [111], the repeat distance for this bulk cell along the $z$ direction is $c=\sqrt{6} a$, and the in-plane NN distances are again compressed or stretched to be equal to $l_{x y} . L_{z}$ is the length along $z$ of the unit cell used for the slab calculations, it includes the vacuum separation between periodic images along $z$. In this way, a plot of surface stress, $\sigma_{x x}^{\text {sulf }}\left(l_{x y}\right)$, versus in-plane lattice constant, $l_{x y}$, was obtained and the in-plane lattice constant at which the surface stress was found to be equal to zero was defined to be the surface atomic diameter, $a^{s}$. From this data, one can also obtain the spring constant $k$ of the bonds within the monolayer (within the harmonic approximation, and considering only NN bonds), by equating $\sigma_{x x}^{\text {sulf }}$ to $\sqrt{3} k\left(s-a^{s}\right) /$ $s$, where $s=a(R h)$ is the NN distance in the substrate. In table 1, we have shown the values thus obtained for the surface atomic diameter, $a^{s}$, the surface stress $\sigma_{x x}^{\text {surf }}$, along with the bulk NN distance $a$ and the spring constant $k$. Note that though $a^{s}$ is indeed smaller than $a$ for both $\mathrm{Ag}$ and $\mathrm{Co}$, this difference does not appear to be large enough for elastic interactions to disfavour the formation of the surface alloys.

\subsection{Surface alloys}

3.4a Structures and formation energy: We first checked the site-dependent stability of surface alloys. We find that site preference is dependent on 
the composition: while, for $x=1.0$ and $x=0.75$, the overlayer atoms prefer to sit at $h c p$ sites, those with $x=0.0,0.25$ and 0.5 prefer to occupy $f c c$ sites. However, the energy differences between $f c c$ and $h c p$ stacking of the overlayers are found to be very small, of the order of $0.1 \mathrm{meV} / \AA^{2}$.

We have calculated the formation energy, $\Delta H$, of the surface alloys (with respect to phase-segregated monolayers of $\mathrm{Ag} / \mathrm{Rh}(111)$ and $\mathrm{Co} / \mathrm{Rh}(111)$ ), as a function of the fractional concentration, $x$, of Co atoms. For a given $x, \Delta H$ for a given overlayer structure is given by:

$$
\begin{aligned}
\Delta H= & E\left(\mathrm{Co}_{x} \mathrm{Ag}_{1-x} / \mathrm{Rh}\right)-x E(\mathrm{Co} / \mathrm{Rh}) \\
& -(1-x) E(\mathrm{Ag} / \mathrm{Rh}),
\end{aligned}
$$

where $E\left(\mathrm{Co}_{x} \mathrm{Ag}_{1-x} / \mathrm{Rh}\right)$ is the total energy of the slab with the alloyed overlayer, and $E(\mathrm{Ag} / \mathrm{Rh})$ and $E(\mathrm{Co} / \mathrm{Rh})$ are the total energies of the slab with phase-segregated monolayers of $\mathrm{Ag}$ and Co respectively. We find that the formation of $\mathrm{Ag}-\mathrm{Co}$ alloys is energetically unfavoured (see table 2 and the plus

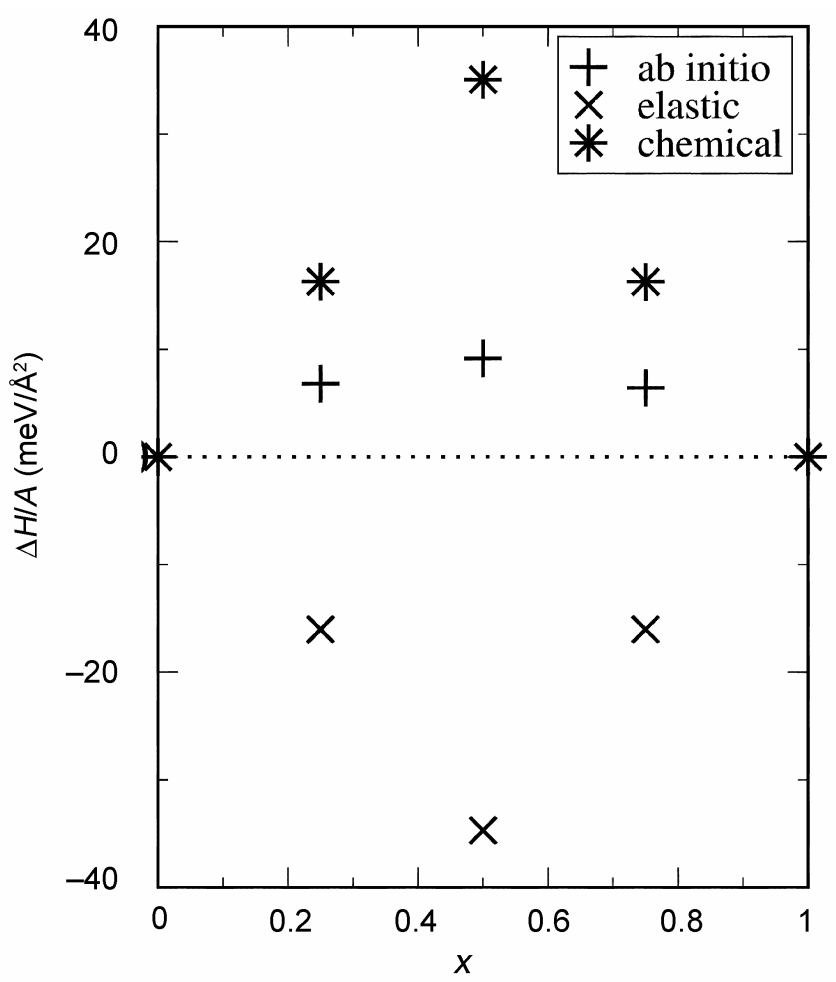

Figure 2. Formation energy of surface alloys as a function of composition: $\triangle H / A$ is the formation energy as given by eq. (2), divided by the surface area $A$, while $x$ is the fractional concentration of $\mathrm{Co}$ in the surface alloy. The plus signs, crosses and asterisks denote the total value, the elastic contribution and the chemical contribution respectively. symbols in figure 2), for all three alloy compositions considered by us. However, we also find that the values of $\Delta H$, though positive (indicating an instability towards phase segregation) are very small. The curve of $\Delta H$ is almost symmetric about the $50 \%$ composition, indicating the dominance of pair-wise interaction terms in the surface alloy.

In order to understand the reason for the positive formation energy of the alloy phases, we have attempted to separate out elastic and chemical effects. We consider an elastic model for our system, in which bonds between all $\mathrm{NN}$ atoms in the overlayer are assumed to be harmonic. The parameters $a^{s}$ and $k$ for $\mathrm{Co}-\mathrm{Co}$ and $\mathrm{Ag}-\mathrm{Ag}$ bonds have been determined for the monolayers of $\mathrm{Ag}$ and $\mathrm{Co}$ on $\mathrm{Rh}(111)$, as described above. For $\mathrm{Ag}-\mathrm{Co}$ bonds, we assume that $a^{s}$ and $k$ are given by the arithmetic mean of the values for $\mathrm{Co}-\mathrm{Co}$ and $\mathrm{Ag}-\mathrm{Ag}$ bonds.

The crosses in figure 2 show our results for the elastic contribution to the heat of formation, determined as described above. Note that this contribu-

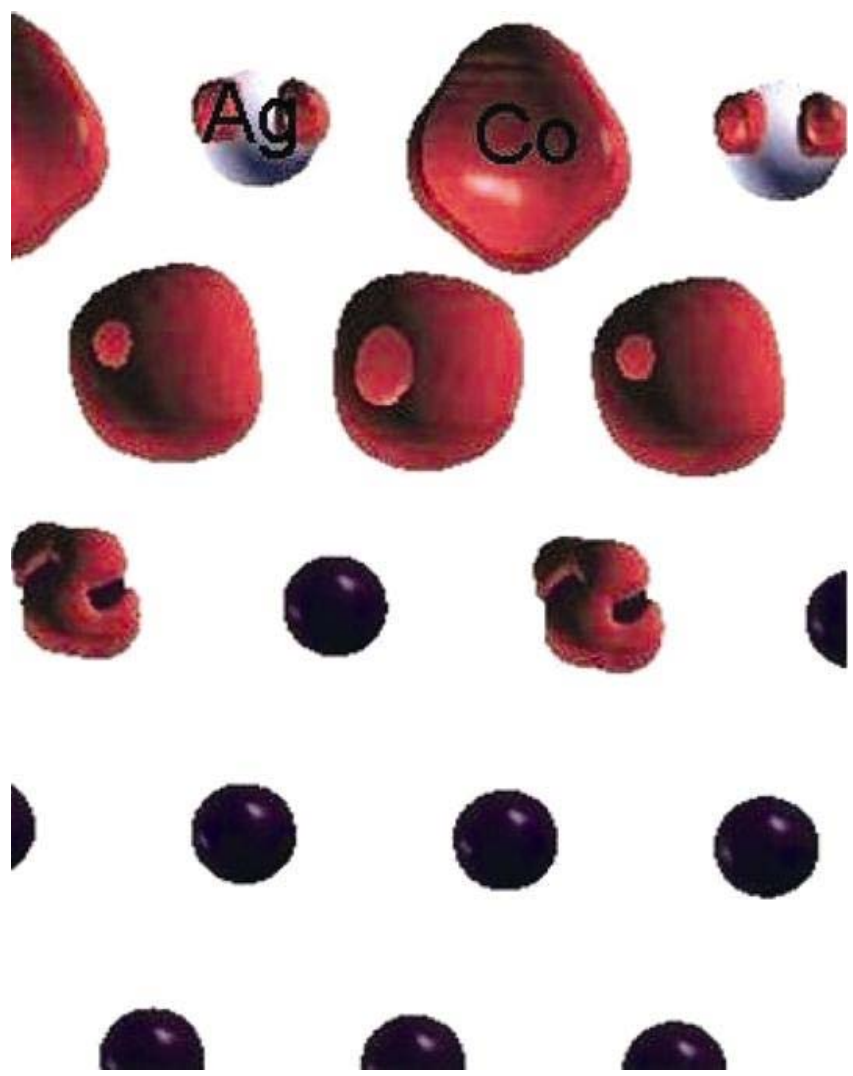

Figure 3. Isosurfaces (in red) of the spin-polarized charge density for $x=0 \cdot 5$. The isosurfaces are drawn for a value of 0.0405 electrons $/ \AA^{3}$. Ag atoms (top layer) are almost unmagnetized while Co atoms (top layer) and the atoms of the top two $\mathrm{Rh}$ layers are considerably magnetized. 
tion is found to be strongly negative, i.e. the elastic contribution to $\Delta H$ always stabilizes the surface alloy. This is in agreement with expectation, given that the average of the values of $a^{s}(\mathrm{Co})$ and $a^{s}(\mathrm{Ag})$ is $2.60 \AA$ which is reasonably close to the substrate lattice constant, and is in agreement with the predictions of Tersoff $f^{5}$ that when elastic interactions are dominant, alloy formation at the surface is always favoured. If we assume that the total value of $\Delta H$ comes from an elastic contribution and a chemical contribution, then the latter is given by the stars in figure 2. Note now that the chemical contribution is strongly positive, i.e. the chemical cost of forming Ag-Co bonds is very high, which disfavours alloying. This is in agreement with the finding that $\mathrm{Ag}$ and Co do not tend to form bulk alloys, and is also in agreement with the conclusions drawn by previous authors (using slightly different arguments) for the closely similar $\mathrm{Ag}-\mathrm{Co} / \mathrm{Ru}(0001)$ system. ${ }^{10}$

We also find that the surface alloys exhibit strong buckling, with the larger Ag atoms pushed outwards, and the smaller Co atoms lying closer to the substrate. The magnitude of the buckling is close to that expected from our values of $a^{s}$, using a hard-sphere model.

3.4b Magnetic behaviour: The magnetic behaviour of this system is also of great interest: will the magnetism increase or decrease upon alloying? In table 2, we have given calculated magnetic moments, $M_{t o t}$, for different values of $x$. Here, $M_{t o t}$ is the total magnetic moment (in $\mu_{B}$ ) per Co atom; this includes induced moments on other atoms. We find that for the Co monolayer on $\mathrm{Rh}(111)$, the magnetic moment is $2.67 \mu_{B}$ per Co atom, which constitutes a huge en-

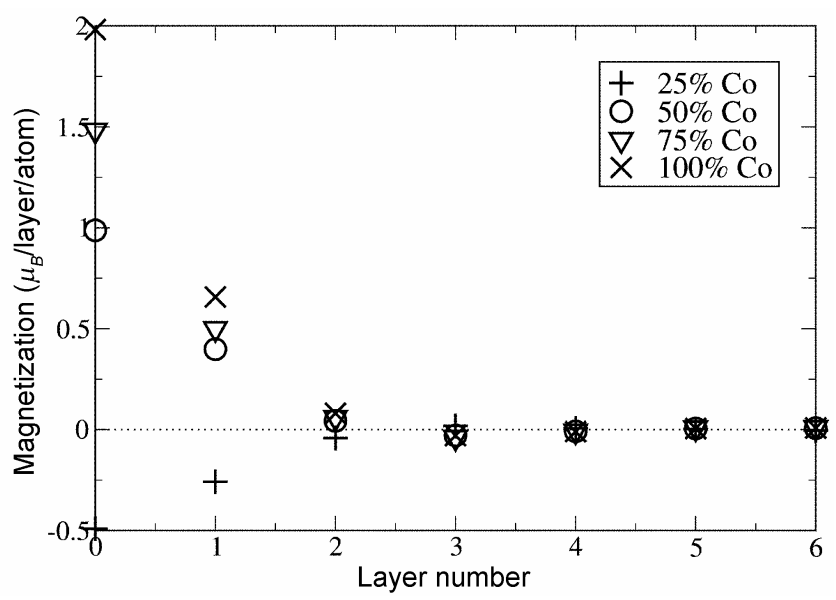

Figure 4. Layer-dependent variation of magnetization. ' 0 ' denotes the surface alloy, while ' 1 ' to ' 6 ' denote the substrate Rh layers. hancement relative to the value for bulk Co $\left(1.71 \mu_{B}\right.$ per Co atom). This enhancement can be attributed to the effect of reduced coordination and lower dimensionality.

We find an overall trend: as the Co concentration $x$ increases, $M_{t o t}$ decreases. The only slight exception to this trend is case of $x=0.75$; this can be attributed to the fact that in this case, the overlayer atoms are found to preferentially occupy $h c p$ sites, and we find that all $h c p$ site alloys are higher in magnetic moment by about $0 \cdot 2 \mu_{B}$ per Co atom from the corresponding $f c c$ site alloys. Also, we find appreciable magnetic moments are induced on the substrate $\mathrm{Rh}$ atoms. In figure 3 we have plotted an isosurface of the spin-polarized charge density, (i.e. the difference between the spin-up and spin-down charge densities) for the system with $x=0 \cdot 5$. From this figure, it can be seen that there are large moments on the Co atoms in the topmost layer (as expected). Interestingly, the electron density in the neighbourhood of the substrate $\mathrm{Rh}$ atoms is found to be also significantly spin-polarized. In figure 4 , we have plotted how the magnetization varies as one progresses from the overlayer to the inner substrate layers. The magnetization decays noticeably, the oscillatory trend which has been observed in other systems ${ }^{20}$ is only weakly manifested in this system. We note that it is not very surprising that $\mathrm{Rh}$ atoms get spin-polarized in the presence of Co, given that bulk Rh is 'almost' ferromagnetic; similar behaviour has also been observed in other Co-Rh systems. ${ }^{21}$

In an attempt to understand the trends in magnetic moments upon alloying, we also performed nonspin-polarized (NSP) DFT calculations on the alloy systems. This was motivated by the well-known Stoner criterion, ${ }^{22}$ that a large NSP density of states (DOS) at the Fermi level favours ferromagnetism. In addition to the the total NSP density of states, we also computed atom-projected densities of states. In table 2, we have given values thus obtained for $M_{C o}$, the magnetic moment of each Co atom in the overlayer, and $D O S_{C o}\left(E_{f}\right)$, the NSP DOS at the Fermi level for Co atoms, as well as the corresponding quantities for the total system. Interestingly, we find that $M_{C o}$ and $M_{t o t}$ exhibit opposite trends: as the Co concentration $x$ increases, $M_{C o}$ increases while $M_{t o t}$ decreases. The corresponding densities of states $D O S_{C o}\left(E_{f}\right)$ and $D O S_{t o t}\left(E_{f}\right)$ roughly follow the same trend, showing that the variation in magnetic moment can be explained, more or less, by the Stoner argument. However, though the variations in magnetic moments and $\operatorname{DOS}\left(E_{f}\right)$ correlate in the expected 
way, the direction of this variation is surprising when considering the Co atoms alone.

\section{Summary and conclusions}

In this paper, we have examined the stability of mixed alloy phases and magnetic properties of surface alloys of $\mathrm{Co}$ and $\mathrm{Ag}$ on a $\mathrm{Rh}(111)$ substrate. Though elastic considerations would seem to favour the formation of this surface alloy, we find that there is a weak but nevertheless systematic tendency for the system to phase-segregate into $\mathrm{Co} / \mathrm{Rh}(111)$ and $\mathrm{Ag} / \mathrm{Rh}(111)$. This is in keeping with a similar finding on the closely-related system of $\mathrm{Co}-\mathrm{Ag} / \mathrm{Ru}(0001)$. Atomic sizes at the surface are found to be less than in the bulk. On separating the heat of formation of the surface alloy into elastic and chemical terms, we find that the former favours alloying, whereas the latter opposes it. The magnetic moment on each $\mathrm{Co}$ atom increases with increasing $\mathrm{Co}$ concentration. However, if one also takes into account the moments induced on other elements, then the opposite trend is seen. Both these trends are in accordance with the Stoner argument.

While we have used relatively small unit cells, and hence only a few compositions, to verify whether or not atomic-level mixing is favoured in this system, we are reasonably confident that calculations on larger unit cells will support the preliminary conclusions presented above, especially since previous calculations on related systems show a fairly smooth variation of formation energy with composition.

In conclusion, we say that size effects alone cannot be used to predict surface alloying, and chemical effects can also play a crucial role in determining formation energies. Further, trends in magnetic moments can be reversed upon also including induced moments.

\section{Acknowledgements}

Helpful discussions with Sylvie Rousset, Vincent Repain and Yann Girard are gratefully acknowl- edged. Funding was provided by the Indo-French Centre for the Promotion of Advanced Research. Computational facilities were provided by the Centre for Computational Materials Science at JNCASR.

\section{References}

1. Chambliss D D, Wilson R J and Chiang S 1991 Phys. Rev. Lett. 661721

2. Nielsen L P, Besenbacher F, Stensgaard I, Laegsgaard E, Engdahl C, Stoltze P, Jacobsen K W and Nørskov J K 1993 Phys. Rev. Lett. 71754

3. Nielsen L P, Besenbacher F, Stensgaard I, Laegsgaard E, Engdahl C, Stoltze P and Nørskov J K 1995 Phys. Rev. Lett. 741159

4. Sprunger P T, Laegsgaard E and Besenbacher F 1996 Phys. Rev. B54 8163

5. Tersoff J 1995 Phys. Rev. Lett. 74434

6. Hwang R Q 1996 Phys. Rev. Lett. 764757

7. Hwang R Q Bartelt M C 1997 Chem. Rev. 971063

8. Sadigh B, Asta M, Ozolins V, Schmid A K, Bartelt N C, Quong A A and Hwang R Q 1999 Phys. Rev. Lett. 831379

9. Thayer G E, Ozolins V, Schmid A K, Bartelt N C, Asta M, Hoyt J J, Chiang S and Hwang R Q 2001 Phys. Rev. Lett. 86660

10. Thayer G E, Bartelt N C, Ozolins V, Schmid A K, Chiang S and Hwang R Q 2002 Phys. Rev. Lett. 89 036101

11. Hohenberg P and Kohn W 1964 Phys. Rev. B136 864

12. Vanderbilt D 1990 Phys. Rev. B41 7892

13. Perdew J P, Burke K and Ernzerhof M 1996 Phys. Rev. Lett. 773865

14. Methfessel M and Paxton A T 1989 Phys. Rev. B40 3616

15. Feynman R P 1939 Phys. Rev. 56340

16. Birch F 1947 Phys. Rev. 71809

17. Ashcroft N W and Mermin N D Mermin 2001 Solid State Physics (Harcourt Asia)

18. Kittel C 2001 Introduction to solid state physics (John Wiley), Seventh ed.

19. Kwon S K, Nabi Z, Kadas K, Vitos L, Johansson B and Ahuja R 2005 Phys. Rev. B72 235423

20. Dennler S, Hafner J, Marsman M and Morillo J 2005 Phys. Rev. B71 094433

21. Harp G R, Parkin S S P, O'Brien W L and Tonner B P 1995 Phys. Rev. B51 12037

22. Stoner E C 1939 Proc. R. Soc. London Ser. A169 339 